\title{
ALEXANDRIA
}

Revista de Educação em Ciência e Tecnologia

ALEXANDRIA

\section{A Argumentação e a Capacidade de Resolver Problemas em Estudantes do Ensino Fundamental}

\author{
Argumentation and Problem-Solving Ability of Elementary School \\ Students
}

\section{Raquel Ruppenthal $^{\mathrm{a}}$; Maria Rosa Chitolina Schetinger ${ }^{\mathrm{a}}$}

a Departamento de Bioquímica e Biologia Molecular, Universidade Federal de Santa Maria, Santa Maria, Brasil rkruppenthal@gmail.com,mariachitolina@gmail.com

Palavras-chave:

Ensino de ciências.

Argumentação.

Habilidades científicas.

Problemas. Ensino

fundamental.
Resumo: Este trabalho objetivou descrever como estudantes do $8^{\circ}$ ano do Ensinc Fundamental resolvem problemas, partindo da hipótese inicial que a capacidad argumentativa dos estudantes tem influência sobre a capacidade de resolução d€ problemas. Para a coleta de dados, utilizou-se um teste da capacidade de resolves problemas e uma entrevista. Na amostra pesquisada, a medida que os estudantes apresentam mais elementos que constituem um argumento em seus enunciados, el consegue atingir melhores níveis no teste de resolução de problemas. Constataram-s $\epsilon$ dificuldades de leitura e síntese, o que pode levar a fragilidades no conhecimento $\epsilon$ aplicação de diversas habilidades científicas. Apresenta-se como perspectivas c produção de atividades didáticas que fomentem o desenvolvimento das habilidades científicas e de leitura.

\begin{abstract}
This study aimed to describe how students of the 8th grade of elementary school solve problems, based on the initial assumption that the argumentative capacity of students have influence on problem solving ability. We used a test's ability to solve problems and an interview for data collection with students of the 8th grade of elementary school. In the studied sample, as students have more elements that constitute an argument in its statements, it can achieve better levels in the problem-solving test. It was found reading difficulties and synthesis, which could lead to weaknesses in the understanding and application of various scientific skills. It presents as prospects the production of educational activities that promote the development of scientific skills and reading.
\end{abstract}




\section{Introdução}

O ensino de ciências vem passando por transformações nos últimos anos. Se no passado, ele visava transmitir informações, hoje volta-se para a preparação do indivíduo consciente, crítico e cidadão (VILANOVA, 2015). A consciência, a criticidade e as competências básicas de aprender a conhecer, aprender a fazer, aprender a conviver e aprender a ser (DELORS, 1998) apenas serão desenvolvidas por meio de um ensino que faça o aluno um protagonista durante o processo de ensino-aprendizagem. Assim, em meados do século XX, surgem iniciativas para tornar o ensino mais ativo, tais como a Aprendizagem Baseada em Problemas (CACHAPUZ et al., 2011; POZO; CRESPO, 2009), os Projetos de Ensino (HERNÁNDEZ; VENTURA, 1998) e mais recentemente, a utilização das Sequências Didáticas Investigativas (MOTOKANE, 2015; SASSERON, 2015). As três abordagens anteriormente citadas pressupõem que o conhecimento é construído mediante o enfrentamento de problemas ou dúvidas, de forma que se desenvolvam conhecimentos conceituais, mas também procedimentais e atitudinais (ZABALA; ARNAU, 2010; POZO 2009).

Da mesma forma, a argumentação é outra linha de pesquisa consolidada, cujos resultados tem-se mostrado interessantes para o ensino de ciências. A argumentação é um gênero típico da linguagem científica, uma vez que no meio científico, é necessário provar o conhecimento com evidências e estabelecer relações entre fatos e conclusões (SASSERON, 2015). Assim, nota-se que existem relações entre a resolução de problemas e a argumentação. No entanto, apesar de inúmeros trabalhos referentes a metodologias de ensino baseadas na resolução de problemas (SALVADOR et al. 2014; MENDES et al., 2012; SOUZA; BASTOS, 2006) e relativos a argumentação (MENDONÇA; JUSTI, 2013; HENAO; STIPCICH, 2008 ;I BRAIM et al. 2013), verificou-se ausência de trabalhos que pesquisem a resolução de problemas e a argumentação conjuntamente, seja analisando os efeitos da capacidade argumentativa sobre a resolução de problemas ou sobre as estratégias utilizadas por estudantes durante o processo.

Assim, o presente trabalho objetivou descrever como estudantes do $8^{\circ}$ ano do Ensino Fundamental resolvem problemas. Partiu-se da hipótese inicial de que a capacidade argumentativa dos estudantes tem influência sobre a capacidade de resolução de problemas. Ou seja, se o estudante consegue utilizar afirmações e justificativas para sustentar suas conclusões, o mesmo apresentará facilidade para resolver problemas que lhe são apresentados.

\section{Revisão bibliográfica}

Conforme Cachapuz et al. (2011, p. 73), “as concepções de ciência que os professores possuem tem implicações no modo como a ensinam". Essas concepções, por sua vez, acabam por orientar as decisões sobre o que ensinar; por que ensinar; qual a melhor maneira de 
ensinar, e como avaliar se os estudantes aprenderam ou se apenas decoraram. Essas questões, não necessariamente nessa ordem, acabam por orientar o professor na organização do seu planejamento, pois ensinar ciências exige reflexão, e passa por diversas decisões.

O planejamento é uma das etapas essenciais para o alcance das metas que se tem no ensino. As metas da educação em ciências tem-se modificado ao longo dos anos. É esperado que

ao concluir o ensino médio, o aluno esteja alfabetizado e, portanto, além de compreender os conceitos básicos da disciplina, seja capaz de pensar independentemente, adquirir e avaliar informações, aplicando seus conhecimentos na vida diária (KRASILCHIK, 2008, p. 12).

Porém, esse trabalho tem início no Ensino Fundamental, pois este representa a base para o Ensino Médio. Portanto, é nessa etapa da escolaridade que se deve iniciar o indivíduo na aprendizagem real de ciências. Entenda-se por aprendizagem real de ciências, aquela que está preocupada em formar cidadãos que sejam responsáveis por seus atos, conscientes, ativos e solidários para a qualidade de vida, além de críticos e atuantes diante daqueles que tomam decisões (KRASILCHIK; MARANDINO, 2007).

Não se alcança a alfabetização científica com práticas educativas voltadas apenas para a transmissão de conceitos. Conhecer o vocabulário da Ciência é importante, mas é a aplicação desse conhecimento em situações reais, ao realizar escolhas ou compreender fenômenos, processos, produtos ou problemas do cotidiano que se verifica a Alfabetização Científica, da mesma forma que colabora por difundir uma imagem de ciência mais realista. Assim, práticas de ensino que se fundamentam apenas no ensino conceitual acabam por defender uma visão da ciência como um produto, estático e acabado (FURMAN, 2009).

Se o conhecimento científico for compreendido como um jogo de hipóteses, expectativas, teorias e observações em constante movimento, que ocorre através da discussão, argumentação e contra-argumentação (CACHAPUZ et al., 2011) percebe-se antes que a Ciência é um processo dinâmico que está em constante transformação (FURMAN, 2009). Por outro lado, como afirma Furman (2009), a ciência entendida como produto ou como processo são faces da mesma moeda, não sendo possível separar uma da outra. No entanto, o que se observa é a prevalência do ensino de ciências como um produto, pronto para o consumo.

Nos últimos anos, estudos vêm sendo realizados em torno de estratégias de ensino baseada na resolução de problemas (SILVÉRIO; MAESTRELI, 2013; MENDES et al. 2012; FRANCISCO JUNIOR et al. 2008; GONZALES; GATTICA, 2008), e mais recentemente, em sequências didáticas investigativas (MOTOKANE, 2015; SASSERON, 2015). Ambas permitem desenvolver os conhecimentos procedimentais além de promover uma visão da ciência como processo. Os conhecimentos procedimentais se referem a habilidades ou saberes 
utilizados para alcançar um objetivo (ZABALA; ARNAU, 2010). Sasseron e Carvalho (2011) consideram que essas habilidades podem ser utilizadas como indicadores da alfabetização científica. Também afirmam que:

\begin{abstract}
Estes indicadores representam ações e habilidades utilizadas durante a resolução de um problema. Alguns destes indicadores estão associados ao trabalho para a obtenção de dados, é o caso do levantamento e do teste de hipóteses em relação a uma situação qualquer; há outros indicadores ligados ao trabalho com estes dados para a classificação, seriação e organização das informações obtidas; também são indicadores da AC: a construção de uma explicação, o uso de justificativa para fundamentar uma ideia e o estabelecimento de previsão sobre o que pode decorrer desta situação; por fim, outros indicadores estão ligados mais diretamente a dimensões epistemológicas da construção do conhecimento, é o caso do uso do raciocínio lógico e do raciocínio proporcional como formas de organizar as ideias que se estão a construir (SASSERON; CARVALHO, 2011, p. 102).
\end{abstract}

As habilidades são um conhecimento de caráter procedimental (POZO; CRESPO, 2009; FURMAN, 2009), um conjunto de ações que serve para alcançar um objetivo (ZABALA; ARNAU, 2010). O conhecimento das habilidades científicas é importante, pois vai além de sua aplicação na resolução de atividades escolares. Estes são saberes básicos para viver na atualidade, onde somos expostos a um número maior de informações a cada dia. Além disso, também são estratégias úteis para a tomada de decisões nas mais diversas situações, para assumir posicionamentos ou para sustentar uma opinião. Da mesma forma, essas habilidades são importantes para compreender como se faz Ciência. Porém, essas habilidades não se desenvolvem espontaneamente, é necessário aprendê-las (FURMAN, 2009; POZO; CRESPO, 2009; POZO et al., 1998).

Lipman (2008, p. 57) afirma que as escolas deveriam ensinar a pensar nas diferentes disciplinas, de maneira crítica e criativa e também com autonomia, de forma a constituir um pensamento de ordem superior. E vai além, afirmando que o desenvolvimento do pensamento superior advém da "capacidade de presumir, supor, comparar, inferir, contrastar ou julgar, para deduzir ou induzir, classificar, descrever, definir ou explicar" (LIPMAN, 2008, p. 57). Estas habilidades podem muito bem ser comparadas com as habilidades científicas descritas anteriormente.

E o qual a relação dessas habilidades com a argumentação? Conforme Sasseron (2015) "a construção de argumentos deflagra a busca por entendimento, validação e aceitação de proposições e processos de investigação em que justificativas e condições de contorno e de refutação precisam ser explicitadas" (SASSERON, 2015, p. 60). E estas são obtidas por meio da realização das habilidades científicas, que são as ações pelas quais o sujeito soluciona situações e através das quais também constrói seu modo de pensar, ou no caso de necessitar comunicar uma resposta, será o estruturador do seu enunciado. 
Dessa forma, delimitar um problema, perceber a interferência de variáveis, elaborar e testar hipóteses, analisar dados para organizar resultados, ao confrontar informações, buscar explicações, são habilidades científicas além de ações argumentativas, importantes tanto para a comunicação ou expressão de ideias como para a resolução de problemas. Por isso, se um indivíduo consegue argumentar utilizando essas habilidades como base na construção de explicações, ele deveria conseguir resolver problemas e situações diversas com facilidade, uma vez que a solução de problemas demanda a utilização dessas mesmas habilidades.

\section{Metodologia}

Para alcançar o objetivo de descrever o processo de resolução de problemas e se o nível argumentativo tem relação com esse processo, optou-se por utilizar as técnicas da pesquisa descritiva, com abordagem qualitativa (OLIVEIRA, 2012). Como instrumentos para a coleta dedados, utilizaram-se um teste de múltipla escolha, e para obter dados sobre o processo de resolução desse teste e descrever o nível argumentativo, elaborou-se uma entrevista. A entrevista (Anexo 1) foi elaborada em conformidade com as estratégias ou habilidades requeridas durante a resolução do teste, de forma a obter dados que pudessem auxiliar na descrição de como os estudantes resolvem problemas. A fim de retratar o nível de argumentação desses estudantes, analisou-se a resposta de uma das questões da entrevista.

O teste da capacidade de solucionar problemas foi traduzido e adaptado de García García e Renteria Rodrigues (2012) e foi utilizado em um teste-piloto (RUPPENTHAL et al., 2015) para verificar sua adequação a estudantes do Ensino Fundamental. Esse teste é composto por 16 questões de múltipla escolha, cada uma verificando uma habilidade requerida para resolver problemas. A partir do número de acertos, é possível verificar o nível de resolução de problemas (Insuficiente, Deficiente, Bom, Muito bom e Excelente).

O teste foi aplicado com 18 estudantes, do $8^{\circ}$ ano do Ensino Fundamental de uma escola pública estadual, na cidade de Ibirubá - RS. O município localiza-se na mesorregião Noroeste Rio-Grandense, tem sua economia baseada nas atividades agrícola e industrial e apresenta o IDH DE 0,765 (ATLAS BRASIL, 2013). Em relação à escola, esta situa-se no centro da cidade e atende alunos do meio rural e urbano. A partir dos resultados do teste selecionaram-se estudantes para a realização da entrevista, utilizando os seguintes critérios: abarcar estudantes de cada um dos níveis da capacidade de resolver problemas, representação de ambos os gêneros e disponibilidade para realizar a entrevista no contra-turno. Assim, compôs-se uma amostra com 11 estudantes, conforme a tabela 1. Como nenhum estudante atingiu o nível Excelente não há nenhum indivíduo dessa categoria na amostra. A entrevista foi realizada no contra-turno a fim de não retirar os estudantes das aulas e causar transtornos aos professores e alunos. 
Tabela 1- Composição da amostra para a entrevista

\begin{tabular}{l|l|l}
\hline \multirow{2}{*}{ Nível de resolução de problemas } & Estudantes & Meninas \\
\cline { 2 - 3 } & Meninos & I 14 \\
\hline Insuficiente & I5 & - \\
\hline Deficiente & I8 & I $4 ;$ I 13 \\
\hline Bom & I 16 & I $2 ;$ I $3 ;$ I $9 ;$ I12 \\
\hline Muito bom & I 11 & \\
\hline
\end{tabular}

Fonte: Autoria própria.

A entrevista foi realizada individualmente na biblioteca da escola, por ser um espaço disponível da escola no contra-turno e pelo fato de ser um ambiente conhecido dos estudantes. Essa entrevista buscou dados para descrever o processo utilizado pelos estudantes durante a resolução do teste. A fim de indicar o nível de argumentação, apresentou-se um problema para os estudantes. Estes deveriam indicar como resolveriam o mesmo. A partir das soluções apontadas, realizou-se a análise conforme instrumento elaborado em trabalho anterior (em avaliação), de acordo com a utilização de afirmações (A), justificativas (J) e conclusões (C) nas respostas. O nível mais simples são aquelas respostas que estão organizadas apenas em torno de afirmações (A); o nível intermediário são as respostas que fazem o uso combinado de afirmações e justificativas $(A+J)$, ou afirmações e conclusões $(A+C)$; enquanto as respostas organizadas em torno de afirmações, justificativas e conclusões $(A+J+C)$ constituem um nível mais elevado. Na tabela 2 apresenta-se um exemplo para cada uma das categorias, elaborada a partir das respostas dadas à questão 9 da entrevista.

Tabela 2 - Níveis de argumentação.

\begin{tabular}{l|l}
\hline Categoria de argumentação & Exemplo \\
\hline Afirmativa (A) & A marca mais conhecida \\
\hline Afirmativa (A) + Justificativa (J) & Olhar o rótulo e ver o que diz \\
\hline Afirmativa (A) + Conclusão ( C ) & Testar para ver se elimina mesmo \\
\hline Afirmativa (A) + Justificativa (J)+ Conclusão (C) & $\begin{array}{l}\text { Levar os dois, usar por uma semana e ver qual é } \\
\text { o melhor }\end{array}$ \\
\hline
\end{tabular}

Fonte: Dados da pesquisa

O intervalo entre a realização do teste e a entrevista foi de 4 semanas, pois foi o tempo necessário para a análise dos resultados do teste-piloto e para verificar a adequação da entrevista aos objetivos deste trabalho. Durante a entrevista, o teste foi disponibilizado para o aluno visualizar as questões e respostas dadas. Cada entrevista durou entre 10 e 20 minutos e as respostas foram registradas e transcritas para a análise, cujos resultados serão apresentados na sequência.

\section{Resultados e discussão}

A partir da análise das respostas à nona questão da entrevista, traçou-se o perfil argumentativo dos estudantes considerando a utilização de afirmações, justificativas e 
conclusões. Os estudantes poderiam indicar mais de uma solução, no entanto, para a análise utilizou-se a solução que atingisse o maior nível argumentativo. Os resultados dessa análise estão indicados na Tabela 3. Observou-se que a maioria das soluções indicadas fica em torno de afirmações com justificativas. A categorização da capacidade de resolver problemas foi obtida conforme o número de acertos do teste e também está indicada na Tabela 2.

Dando sequência ao trabalho, para obter uma visão geral de como os estudantes resolveram o teste, estes foram questionados sobre como resolveram as questões. Houve unanimidade em torno das estratégias de leitura e interpretação. No entanto a medida que a capacidade de resolver problemas melhorava, verificava-se a utilização de outras estratégias, tais como pensar sobre as perguntas, usar a lógica, buscar e anotar informações importantes, relacionar afirmativas das questões com as respostas e destacar pontos principais, conforme a Tabela 3.

Tabela 3 - Resultados do nível argumentativo, capacidade de resolver problemas e estratégias utilizadas para responder ao questionário.

\begin{tabular}{|c|c|c|c|}
\hline ALUNO & $\begin{array}{l}\text { NÍVEL } \\
\text { ARGUMENTATIVO }\end{array}$ & $\begin{array}{l}\text { CAPACIDADE DE } \\
\text { RESOLVER } \\
\text { PROBLEMAS }\end{array}$ & $\begin{array}{l}\text { Como você resolveu aos problemas do } \\
\text { questionário? }\end{array}$ \\
\hline 5 & $\mathrm{~A}+\mathrm{J}$ & \multirow[t]{2}{*}{ Insuficiente } & $\begin{array}{l}\text { Lia bem, resolver na cabeça (ver qual a melhor } \\
\text { resposta). }\end{array}$ \\
\hline 14 & $\mathrm{~A}+\mathrm{J}$ & & $\begin{array}{l}\text { Leu e interpretou. Difícil porque não interpreta } \\
\text { bem os enunciados. }\end{array}$ \\
\hline 8 & & Deficiente & Não compareceu. \\
\hline 4 & $\mathrm{~A}+\mathrm{J}$ & \multirow[t]{3}{*}{ Bom } & Leu e interpretou. \\
\hline 13 & $\mathrm{~A}$ & & Leu e interpretou. \\
\hline 16 & A & & $\begin{array}{l}\text { Leu, pensou sobre as perguntas, } \\
\text { desenvolvendo o pensamento e respondeu. }\end{array}$ \\
\hline 2 & $\mathrm{~A}+\mathrm{J}+\mathrm{C}$ & \multirow[t]{5}{*}{ Muito bom } & $\begin{array}{l}\text { Usando lógica, que é um pensamento } \\
\text { combinar com outro. Leu várias vezes } \\
\text { buscando informações. }\end{array}$ \\
\hline 3 & $\mathrm{~A}$ & & $\begin{array}{l}\text { Ler anotando o mais importante. Usar lógica, } \\
\text { raciocínio e cálculos. }\end{array}$ \\
\hline 9 & $\mathrm{~A}+\mathrm{J}+\mathrm{C}$ & & $\begin{array}{l}\text { Leu, entendeu. Relacionou afirmativas dos } \\
\text { textos com as respostas. }\end{array}$ \\
\hline 11 & $\mathrm{~A}+\mathrm{C}$ & & Leu, interpretou e marcou. \\
\hline 12 & $\mathrm{~A}+\mathrm{J}$ & & $\begin{array}{l}\text { Usando lógica. Lê, vê os pontos principais e } \\
\text { resolve. }\end{array}$ \\
\hline
\end{tabular}

Fonte: Dados da pesquisa

Preparar estudantes para resolver problemas é uma das metas do ensino de ciências (CACHAPUZ et al., 2011; POZO; CRESPO, 2009; POZO et al., 1998), e resolvê-los, por sua vez exige do indivíduo a mobilização de habilidades, estratégias (POZO et al., 1998) também denominados conhecimentos procedimentais (ZABALA; ARNAU, 2010). No teste em questão ou qualquer atividade escolar, o primeiro passo para a solução é a leitura juntamente com a interpretação. A compreensão do problema é essencial, pois é assim que se percebe o que é solicitado e quais as informações que devem ser mobilizadas para a solução (GARCÍA GARCÍA; RENTERIA RODRIGUEZ; 2012). Uma estudante, categorizada com desempenho 
insuficiente no teste, apontou em sua fala que achou difícil resolver o questionário devido a sua dificuldade em interpretar enunciados, ou ordens de questões. Essa fala corrobora o anteriormente exposto e sinaliza para a importância de se dar atenção a esse aspecto durante as aulas.

Um estudante categorizado como Bom, afirma que "Leu, pensou sobre as perguntas, desenvolveu o pensamento e respondeu". Percebe-se que o mesmo fundamentou suas escolhas a partir da reflexão sobre sua interpretação, demonstrando a capacidade de metacognição, ou seja, pensar o processo de resolução dos problemas além de conseguir verbalizar o processo, o que demonstra que o mesmo apresentou consciência do processo empregado. Conforme García García e Renteria Rodriguez (2012) a habilidade de pensar em termos de como agir para solucionar um problema ou atividade é um fator importante na solução de problemas. Para resolver alguma situação, em primeiro lugar realiza-se uma análise da situação, destacando informações relevantes para sua solução. Após essa etapa inicial, o indivíduo revisa os esquemas de atuação dos quais dispõe para resolver a situação e opta por aquele que se mostra mais eficaz, para então, aplicá-lo a situação que lhe foi apresentada (ZABALA; ARNAU, 2010). É necessário considerar que essas etapas todas podem ocorrer inconscientemente, ou seja, os demais estudantes podem ter seguido uma via similar, porém não tiveram consciência disso.

Com exceção do estudante 11, os demais estudantes cuja capacidade de resolver problemas foi Muito bom, foram ainda mais detalhados na descrição da sua maneira de resolver as questões. Além da leitura e interpretação, três falam sobre a utilização da lógica, entendida por eles como o encadeamento/a combinação entre pensamentos. Salienta-se que na rede de ensino pública do município, a Filosofia é um componente curricular a partir do $6^{\circ}$ ano do Ensino Fundamental e aborda questões relativas à ética, moral e também princípios sobre lógica. É possível que esses estudantes tenham se valido desses conhecimentos como uma estratégia ou ferramenta durante a resolução do teste. No entanto, e os livros didáticos de ciências abordam aspectos relativos a organização e sistematização lógica do conhecimento científico?

Cabe aqui refletir sobre o processo utilizado na construção e sistematização do conhecimento científico, ou seja, considerar a epistemologia da Ciência. Para uma teoria ser considerada válida, ela deve ter dados e fatos como suportes, de maneira a provar uma relação causal entre os fatos observados e a conclusão oferecida (CACHAPUZ et al., 2011). Dessa forma, se há uma base lógica para construir a Ciência, a compreensão dela poderia ocorrer de forma similar.No entanto, em que momentos das aulas de ciências se dá atenção a este aspecto? Como os materiais didáticos disponíveis nas escolas contribuem para divulgar essa visão de Ciência? Os textos utilizados como meios para a organização do conhecimento estão 
organizados de forma a mostrar e explicar a estrutura do conhecimento científico? $\mathrm{Ou}$ estimulam a escrita de forma a empregar esta estrutura? $\mathrm{O}$ fato dos alunos afirmarem que utilizaram a lógica merece destaque no sentido de que se repensem as práticas e materiais didáticos, a fim de realmente desenvolver um pensamento lógico científico.

Voltando a questão de como estudantes resolvem problemas, parece evidente que quanto maior o arsenal de estratégias de que o estudante possui e quanto maior a sua consciência em relação ao processo de solução, maior é a chance de conseguir resolver situações problemáticas com êxito.

Após obter essa visão mais geral sobre o processo empregado pelos estudantes para resolver o teste, prosseguiu-se a entrevista para investigar as habilidades mais específicas relativas à solução de problemas. Essas habilidades foram investigadas a partir de questões abertas e fechadas e também na forma de afirmativas, com uma escala de cinco níveis. A análise dos resultados será realizada por fator/indicador de resolução de problemas.

Para García García e Renteria Rodrigues (2012), o fator mais importante para resolver problemas é a capacidade de predição e transferência, verificável através da seleção de hipóteses adequadas, identificação da melhor solução bem como pela determinação de situações em que a solução apontada é aplicável. Dos estudantes entrevistados, seis afirmam saber o que é uma hipótese, afirmando ser uma "ideia que pode ser verdadeira ou não" ou então que seria "uma opção". Alguns indicaram que hipótese é "uma dúvida, ou pergunta". Ou seja, nem todas as definições dadas por eles podem ser consideradas como corretas. Então, apesar da maioria dos estudantes afirmarem que sabe o que é uma hipótese, as definições dadas pelos mesmos indicam o contrário. Conforme Sasseron (2008), é importante que os estudantes conheçam o significado das palavras para que então possam estabelecer relações e construir entendimento relativo ao assunto. Dessa forma, pode-se afirmar que os estudantes pesquisados não compreendem o significado da palavra hipótese para a cultura científica.

No teste de resolução de problemas utilizado, esse fator foi verificado nos três problemas iniciais. Dos estudantes da categoria Insuficiente, não houve nenhum acerto, inclusive da aluna que afirmou saber o que é uma hipótese. Entre os estudantes da categoria Bom, dois afirmaram saber o que hipótese e um que afirmou saber, mas sem muita certeza observou-se que das três questões, cada um acertou apenas uma. Já entre os estudantes da categoria Muito bom, houve dois acertos de três questões, e em relação a definição de hipótese, afirmaram conhecer bem (3) ou mais ou menos (2). Com os dados coletados não há como dizer se há presunção do saber o por parte dos estudantes. No entanto, é um ponto que merece atenção e reflexão por parte dos professores.

Conforme Machado e Sasseron (2012), os indicadores ou habilidades representam ações utilizadas para resolver problemas e situações. O levantamento e teste de hipóteses está 
relacionado a compreensão da situação que está em análise (SASSERON; CARVALHO, 2008), normalmente presente no início de discussões ou atividades investigativas (SASSERON; CARVALHO, 2011). Elaborar uma hipótese ou escolher a mais adequada pressupõe a capacidade de relacionar causas e efeitos, além de perceber a existência de variáveis que podem alterar o efeito esperado. Assim, os resultados obtidos no questionário e nas entrevistas, sugerem que o conceito hipótese, na verdade, é desconhecido tanto em definição quanto em aplicação.

Outros dois fatores importantes para resolver problemas são a Capacidade de síntese e a Leitura crítica (GARCÍA GARCÍA; RENTERIA RODRIGUES, 2012). Cada um deles é constituído por habilidades menores, mas vamos no ater a dois: a seleção de palavras-chave e a seleção de informações relevantes. Estes podem ser equiparados aos indicadores classificação de informações, seriação de informações e organização de informações, apontados por Sasseron e Carvalho (2008) como habilidades relacionadas ao trabalho com dados. Seria a etapa intermediária entre a produção de hipóteses e a elaboração de explicações.

Segundo Garcia Garcia e Renteria Rodriguez (2012), selecionar palavras-chave que resumam um texto é um descritor da capacidade de síntese do indivíduo. Se considerarmos a palavra como o signo que media a construção e formação do pensamento (VIGOTSKY, 2007), percebe-se a importância que a escolha de palavras chave assume para e elaboração conceitual do estudante bem como na reestruturação do seu conhecimento ou então, como importante etapa durante a organização das informações. As palavras-chave assumem importância, pois elas conseguem descrever sinteticamente um tema, assunto, conteúdo ou teoria. Por meio do compartilhamento de seus significados, é possível construir um conhecimento. Dessa forma, ao reduzir um problema em palavras ou ideias-chave, tem-se a possibilidade de iniciar a análise e compreensão do problema.

Por isso, os estudantes foram questionados se sabiam o que é uma palavra-chave. A maioria afirmou conhecer enquanto dois afirmaram reconhecer, porém com certa insegurança. Em relação a realização dessa habilidade, ou seja, a sua execução, sete estudantes afirmaram que conseguem escolher palavras-chave com certa facilidade, enquanto outros três afirmam não apresentar facilidade nem dificuldade. Apesar dessas afirmações observou-se que seis estudantes acertaram a questão do teste que avaliava essa habilidade. Salienta-se que as alternativas do teste forneciam grupos de palavras-chave para que o estudante optasse por uma das alternativas. Essa habilidade não pode ser considerada uma das mais simples, pois envolve a visão geral da situação ou informação, para então, verificar a hierarquia ou níveis de organização entre os conceitos. Esse achado sugere que a capacidade de síntese não é uma 
habilidade sobre a qual os estudantes tenham domínio, o que por sua vez pode comprometer a capacidade de resolver outros problemas, ou mesmo interferir na produção de explicações.

Por outro lado, gráficos e tabelas são uma forma de organizar informações de maneira sintética e, por isso, Garcia Garcia e Renteria Rodriguez (2012) apontam a habilidade de compreender informações de gráficos e tabelas como um fator importante para resolver problemas. Ribas et al. (2012) e Krasilchik (2008) afirmam que gráficos, tabelas e congêneres são típicas formas de linguagem utilizadas para representar informações e conhecimento nas Ciências. Pode-se firmar que a capacidade de compreender e interpretar tabelas e gráficos variados é um atributo importante tanto no cotidiano como para a resolução de problemas.

Dos entrevistados, três afirmaram ter muita facilidade ao interpretar gráficos e tabelas, dois indicaram realizar tarefas do gênero com certa facilidade (um indicativo de que pode ser uma dificuldade) e cinco afirmaram não ter facilidades nem dificuldades neste quesito. Contrastando essas afirmativas com os resultados do teste, observou-se que nas questões específicas (11 e 12) para esse fator, os acertos estão relacionados ao nível da capacidade de resolver problemas, ou seja, quanto maior o nível, maior foi a tendência ao acerto. Ao observar os resultados de todas as questões que envolviam a compreensão de gráficos e tabelas $(7,9,11,12$ e 14) para elaborar explicações ou justificar as escolhas, observa-se praticamente o mesmo: a melhor compreensão de gráficos e tabelas está relacionada com o melhor desempenho no teste de resolução de problemas.

Ler é interagir com o texto (LEFFA, 1996). Antes de efetuar uma leitura crítica, se faz necessário que o estudante seja capaz de realizar uma leitura mais básica, que permita apreender as informações principais e secundárias, para que possa estabelecer relações entre estas e os próprios conhecimentos. A maioria dos entrevistados afirmou que gosta de ler, sendo que lêem livros de suspense, aventura, mistério, ação ou romance. $\mathrm{O}$ fato de possuir o hábito de leitura é importante, pois conforme Lipman (2008) ler é uma super-habilidade que está na base de todas as outras. Pelo hábito de leitura se desenvolvem algumas características, tais como, destacar ideias principais, descrever relações entre personagens, fazer previsões sobre o desfecho da história, fazer resumos, comparar personagens e histórias, entre outros.

Para resolver problemas escolares ou do cotidiano, muitas vezes precisa-se verificar quais informações e variáveis são de fato importantes para a sua resolução (GARCÍA GARCÍA; RENTERIA RODRIGUES, 2012), o que normalmente ocorre através da leitura. Dessa forma, perguntou-se aos entrevistados se eles se sentiam capazes de distinguir informações relevantes das não relevantes ao resolver problemas. Pela primeira vez, estudantes reconheceram apresentar um pouco de dificuldades (três estudantes). A consciência de apresentar dificuldades é um importante ponto de partida, pois permite ao indivíduo buscar maneiras de superar a dificuldade. Quando os alunos foram questionados 
sobre sua capacidade em perceber pontos de vista ou informações semelhantes entre textos, novamente a maioria julgou ter muita facilidade. Essa habilidade, de certa forma, está relacionada ao perfil de leitura individual ou com os processos de leitura que se dão nas aulas de ciências.

Ao reconhecer que a linguagem científica apresenta características que a fazem diferente da linguagem coloquial, por exemplo, e que é marcada por formas discursivas e enunciados que lhe são próprias (MORTIMER et al., 1998; BARGALLÓ, 2005), supõe-se que a leitura de um texto de divulgação científica ocorra de forma diversa daquela que ocorre ao ler um romance. Se a Ciência apresenta uma forma própria de sistematizar os conhecimentos, que no caso das Ciências Naturais é através das proposições de causa e efeito/consequência, para bem ler um texto científico, seja de divulgação ou não, se faz necessário a capacidade de selecionar dados e informações para verificar se a argumentação é convincente, compreender o significado que as palavras assumem naquele contexto de produção (SASSERON, 2008). Portanto, é necessário aprender a ler em ciências, o que pode ter influência na resolução de problemas científicos.

Em relação à abordagem utilizada pelos estudantes para solucionar problemas, nove afirmam que procuram resolver em etapas a fim de facilitar o processo. Essas etapas ditas pelos estudantes, tem relação com a forma como resolveram ou na sequência empregada pelos mesmos: ler, interpretar e resolver. Chama a atenção o único estudante que afirmou ser mais fácil resolver sem dividi-lo em partes, uma vez que "é importante considerar o todo para resolver [o problema]". Talvez seja uma conseqüência da Ciência defendida por Descartes (2001) de que para compreender algo seria necessário dividi-lo em partes tanto quanto fosse possível. No entanto, atualmente há um movimento em torno da reunificação dos saberes para que se possa enfrentar os problemas globais e tornar a Ciência mais ética e humana (MORIN, 2011).

\section{Algumas considerações}

A educação científica está voltada para a formação de indivíduos que utilizem seu conhecimento para viver com qualidade, saúde e para que possam estar prontos as transformações que a vida apresenta e também para resolver problemas. Espera-se que a experiência escolar lhes proporcione conhecimentos que contribuam para isso. Dessa forma, ao descrever como estudantes resolvem problemas escolares, obtêm-se uma visão de aspectos positivos e também frágeis nesse sentido.

Nossa hipótese inicial era de que o nível argumentativo influenciaria a capacidade de resolver problemas, o que foi verificado em parte. Nessa amostra pesquisada, a medida que o estudante consegue introduzir mais elementos em seus enunciados, ele atingiu melhores 
níveis no teste de resolução de problemas. No entanto não é possível generalizar essa afirmação. Pode-se constatar que quanto mais estratégias (procedimentos) o estudante conhece e quando ele apresenta consciência do processo de resolução (capacidade metacognitiva), melhor foi o resultado no teste de resolução de problemas.

No entanto, há aspectos que necessitam maior atenção. No que se refere ao conceito de hipóteses, ficou evidente pelo resultado do questionário e das entrevistas, que o conceito é desconhecido tanto em definição quanto em aplicação. Como seria possível propor alternativas de solução para algum problema ou propor formas de testá-las se o significado e a função da hipótese são desconhecidas? Indo além, como esperar que os estudantes percebam a Ciência como um processo na construção e sistematização do conhecimento? Outra fragilidade encontrada no processo com os alunos foi que a capacidade de síntese não é uma habilidade sobre a qual os estudantes tenham domínio.

Os resultados desse trabalho podem não ser generalizáveis a todos os estratos da população estudantil, uma vez que foram obtidos com uma pequena amostra de estudantes. No entanto indicam a existência de fragilidades referentes ao conhecimento e aplicação de diversas habilidades científicas, bem como aspectos a serem considerados pelo professor ao planejar suas aulas. Sugerem também que a leitura, enquanto habilidade básica, precisa receber maior atenção durante as aulas. Dessa forma, apresenta-se como perspectivas a produção de atividades didáticas que fomentem o desenvolvimento dessas habilidades, além de proporcionar aos professores de educação básica um material específico.

\section{Referências}

ATLAS DO DESENVOLVIMENTO HUMANO NO BRASIL. 2013. Disponível em <http://www.atlasbrasil.org.br/2013/pt/perfil_m/ibiruba_rs\#idh> Acesso em 4 fev. 2015.

BARGALLÓ, C. M.. Aprender ciências a través del lenguaje. Educar, v. 2, p. 27-39, 2005. Disponível em $<$ http://gent.uab.cat/conxitamarquez/sites/gent.uab.cat.conxitamarquez/files/Aprender\%20cienc ias\%20a\%20traves\%20del\%20lenguaje_0.pdf > Último acesso em: dez. 2015.

CACHAPUZ, A.; GIL-PEREZ, D.; CARVALHO, A. M. P.; PRAIA, J.; VILCHES, A. A necessária renovação no ensino de ciências. 2 ed. São Paulo: Cortez, 2011.

DELORS, J. (Coord.). Os quatro pilares da educação. In: Educação: um tesouro a descobrir. São Paulo: Cortez, 1998.

DESCARTES, R. Discurso do método. São Paulo: Martins Fontes, 2001.

FRANCISCO JUNIOR, W. E.; FERREIRA, L. H.; HARTWIG, D. R.. A dinâmica de resolução de problemas: analisando episódios em sala de aula. Ciências e Cognição, v. 1, n. 3, p. 82-99, 2008. 
FURMAN, M. O ensino de Ciências no Ensino Fundamental: colocando as pedras fundacionais do pensamento científico. Instituto Sangari Brasil, 2009.

GARCÍA GARCÍA, J. J.; RENTERIA RODRIGUEZ, E. La medición de la capacidad de resolucion de problemas em las ciências experimentales. Ciência \& Educação, v. 18, n. 4, p . 755-767, 2012.

GONZÁLEZ, J. P. C.; GATICA, M. Q. Resolución de problemas científicos desde La historia de La ciencia: retos y desafios para promover competencias cognitivo lingüísticas em la química escolar. Ciência \& Educação, v. 14, n. 2, p. 197-212, 2008.

HENAO, B. L.; STIPCICH, M. S. Educación em ciencias y argumentación: La perspectiva de Toulmin como possible respuesta as demandas y desafios contemporâneos para La enseñanza de las Ciencias Experimentales. Revista Electronica de Enseñanza de las Ciencias, v.7, n.1, p. 47-62, 2008.

HERNÁNDEZ, F.; VENTURA, M. A organização do currículo por projetos de trabalho. Porto Alegre: Artes Médicas, 1998.

IBRAIM, S. S.; MENDONÇA, P. C. C.; JUSTI, R..Contribuições dos Esquemas Argumentativos de Walton para análise de argumentos no contexto do Ensino de Ciências. Revista Brasileira de Pesquisa em Educação em Ciências, v. 13, n. 1, p. 159-185, 2013.

KRASILCHIK, M. Prática de ensino de biologia. São Paulo: EdUSP, 2008.

KRASILCHIK, M.; MARANDINO, M. Ensino de Ciências e Cidadania. 2.ed. São Paulo: Editora Moderna. 2007

LEFFA, V. J. Aspectos da leitura. Porto Alegre: Sagra: DC Luzzatto, 1996.

LIPMAN, M. O pensar na educação. 4.ed. Rio de Janeiro: Vozes, 2008.

MACHADO, F. V.; SASSERON, L. H. As perguntas em aulas investigativas de Ciências: a construção teórica de categorias. Revista Brasileira de Pesquisa em Educação em Ciências, v. 12, n.2, p. 29-44, 2012.

MENDES, G.; MARTINS, C.; OLIVEIRA, C.; SILVA, M. J.; VILAÇA, S.Contributos da aprendizagem baseada em problemas no desempenho do estudante de enfermagem em ensino clínico. Revista de Formación e Innovación Educativa Universitaria, n. 5, n. 4, p. 227-240, 2012.

MENDONÇA, P. C. C.; JUSTI, R. S. Ensino-Aprendizagem de Ciências e Argumentação: Discussões e Questões Atuais. Revista Brasileira de Pesquisa em Educação em Ciências, v. 13, n.1, p.187-216, 2013.

MORIN, E. Os setes saberes necessários à educação do futuro. São Paulo: Cortez, 2011.

MORTIMER, E. F.; CHAGAS, A. N.; ALVARENGA, V. T. Linguagem científica versus linguagem comum nas respostas escritas de vestibulandos. Investigações em Ensino de Ciências, v.3, n.1, p. 7-19, 1998. 
MOTOKANE, M. T. Sequências didáticas investigativas e argumentação no ensino de ecologia. Revista Ensaio, v.17, n.especial, p. 115-137, 2015.

OLIVEIRA, M. M. Como fazer pesquisa qualitativa. 4.ed. Petrópolis: Vozes, 2012.

POZO, J. I.; CRESPO, M. A. G. A aprendizagem e o ensino de ciências: do conhecimento cotidiano ao conhecimento científico. 5.ed. Porto Alegre: Artes Médicas, 2009.

POZO, J. I.; ECHEVERRÍA, M. P.P.; CASTILLO, J. D.; ANGÓN, Y. P. A solução de problemas: aprender a resolver, resolver para aprender. Porto Alegre: Artes Médicas, 1998.

RIBAS, A.S.; STADLER, R.C.L.; SILVA, S.C.R.; GALVÃO, J.R. A importância da utilização de linguagens para ensinar física no ensino médio. In: STADLER, R. C. L. (Org.) A importância da linguagem no ensino de ciências: experiências e reflexões. Curitiba: Editora CRV, 2012. p. 29-59.

RUPPENTHAL, R.; COUTINHO, C. ORTH, K. M., MEIRA, M. S.; SCHETINGER, M. R. C. A capacidade de resolver problemas: um estudo-piloto sobre a adequação de um teste de desempenho na resolução de problemas. Ciência e Natura, v. 37, n.2, p. 833-848, 2015.

SALVADOR, D. F.; ROLANDO, L. G. R; OLIVEIRA, D. B.; VASCONCELLOS, R. F. R. R. Aplicando os princípios da Aprendizagem Baseada em Problemas como modelo instrucional no contexto de uma feira de ciências. Revista Electrónica de Enseñanza de las Ciencias, v. 13, n. 3, p.292-317, 2014.

SASSERON, L.H. Alfabetização Científica no Ensino Fundamental - Estrutura e indicadores deste processo em sala de aula. Tese de doutorado em Educação - Universidade de São Paulo, São Paulo, 2008.

SASSERON, L. H. Alfabetização científica, ensino por investigação e argumentação: relações entre ciências da natureza e escola. Revista Ensaio, v.17, n.especial, p. 49-67, 2015.

SASSERON, L. H.; CARVALHO, A. M. P. Almejando a alfabetização científica no ensino fundamental: a proposição e a procura de indicadores do processo. Investigações em Ensino de Ciências, v.13, n.3, p.333-352, 2008.

SASSERON, L. H.; CARVALHO, A. M. P.. Construindo argumentação na sala de aula: a presença do ciclo argumentativo, os indicadores de alfabetização científica e o padrão de Toulmin. Ciência \& Educação, v. 17, n. 1, p. 97-114, 2011.

SILVÉRIO, L. E. R.; MAESTRELLI, S. R. P.. Ensinar genética resolvendo problemas: potencial de uma estratégia didática. In: DUSO, L.; HOFFMANN, M.B. (Org.) Docência em Ciências e Biologia: propostas para um continuado (re) iniciar. Ijuí, RS: Editora Unijuí, 2013. p. 175-204.

SOUZA, C. A.; BASTOS, F. P. Um ambiente multimídia e a resolução de problemas de física. Ciência \& Educação, v. 12, n. 3, p. 315-332, 2006.

VIGOTSKY, L. S. A formação social da mente: o desenvolvimento dos processos psicológicos superiores. 7. ed. São Paulo: Martins Fontes, 2007. 
VILANOVA, R. Educação em ciências e cidadania: mudança discursiva e modos de regulação na política do Programa Nacional do Livro Didático. Ciência \& Educação, v. 21, n. 1, p. 177-197, 2015.

ZABALA, A.; ARNAU, L. Como aprender e ensinar competências. Porto Alegre: Artes Médicas, 2010.

\section{ANEXO 1 - Entrevista}

CÓDIGO: IDADE:

1- Como você resolveu os problemas do questionário?

2- Leia as afirmativas abaixo e marque de acordo:

\begin{tabular}{l|l|l|l|l|l} 
Afirmativas & $\begin{array}{l}\text { Com } \\
\text { muita } \\
\text { dificuldade }\end{array}$ & $\begin{array}{l}\text { Com } \\
\text { pouca } \\
\text { dificuldade }\end{array}$ & $\begin{array}{l}\text { Nem } \\
\text { facilidade, } \\
\text { nem } \\
\text { dificuldade }\end{array}$ & $\begin{array}{l}\text { Com } \\
\text { pouca } \\
\text { facilidade }\end{array}$ & $\begin{array}{l}\text { Com } \\
\text { muita } \\
\text { facilidade }\end{array}$ \\
\hline Eu entendo gráficos e tabelas & & & & & \\
\hline $\begin{array}{l}\text { Consigo escolher palavras } \\
\text { chave de textos/conteúdos }\end{array}$ & & & & & \\
\hline $\begin{array}{l}\text { Consigo perceber semelhanças e } \\
\text { diferenças entre textos ou } \\
\text { gráficos }\end{array}$ & & & & & \\
\hline $\begin{array}{l}\text { Sei distinguir entre informações } \\
\text { importantes ou não para resolver } \\
\text { problemas }\end{array}$ & & & & & \\
\hline Consigo interpretar textos & & & & & \\
\hline Consigo justificar respostas & & & & & \\
\hline
\end{tabular}

3- Você sabe o que é hipótese?
( ) SIM ( ) NÃO
) MAIS OU MENOS

4- Você sabe o que são palavras chave?
( ) SIM ( ) NÃO ( ) MAIS OU MENOS

5- Você lê bastante?

\section{( ) SIM ( ) NÃO ( ) MAIS OU MENOS}

6- Que tipo de leitura?

7- Quando você está resolvendo problemas, resolve em partes ou tudo ao mesmo tempo?

8- Quando você está resolvendo algum tipo de problema, consegue saber o que é necessário saber para resolver ele?

9- Duas fábricas de sabonetes que são concorrentes estão veiculando comerciais na TV afirmando que são os melhores. Como argumento, dizem que eliminam 99,9\% dos microorganismos das mãos. Sabendo que os sabonetes das duas marcas têm os mesmos preços e que o tamanho/peso deles é igual, como você escolheria qual deles comprar? 
Tabela indicando as questões que os estudantes acertaram na solução do questionário.

\begin{tabular}{|c|c|c|c|c|c|c|c|c|c|c|c|c|c|c|c|c|c|c|c|}
\hline & & \multicolumn{3}{|c|}{$\begin{array}{r}\text { FATOR } \\
1\end{array}$} & \multicolumn{3}{|c|}{$\begin{array}{r}\text { FATOR } \\
2\end{array}$} & \multicolumn{2}{|c|}{ FAT.3 } & \multicolumn{2}{|c|}{ FAT.4 } & \multicolumn{2}{|c|}{ FAT.5 } & \multicolumn{3}{|c|}{ FATOR 6} & \multirow{2}{*}{\begin{tabular}{|l|} 
F7 \\
16
\end{tabular}} & \multirow[b]{3}{*}{ ACERTOS } & \multirow{3}{*}{$\begin{array}{c}\text { CATEGORIA } \\
\text { NOMINAL }\end{array}$} \\
\hline & GÊNERO & 1 & 2 & 3 & 4 & 5 & 6 & 7 & 8 & 9 & 10 & 11 & 12 & 13 & 14 & 15 & & & \\
\hline & & $\mathbf{B}$ & $\begin{array}{l}\mathbf{D} \\
\text {. }\end{array}$ & $\mathbf{A}$ & $\mathbf{C}$ & \begin{tabular}{l|l}
$\mathbf{A}$ \\
\end{tabular} & B & B & $\mathbf{A}$ & $\mathbf{D}$ & $\mathbf{D}$ & $\mathbf{C}$ & $\mathbf{C}$ & $\mathbf{D}$ & $\mathbf{C}$ & B & $\mathbf{A}$ & & \\
\hline 5 & M & $\mathrm{D}$ & $\mathrm{C}$ & $\mathrm{C}$ & $\mathrm{C}$ & $\mathrm{D}$ & $\mathrm{A}$ & B & A & A & $\mathrm{C}$ & B & B & B & B & $\mathrm{C}$ & B & 3 & INSUFICIENTE \\
\hline 14 & $\mathrm{~F}$ & $\mathrm{~A}$ & $\mathrm{~B}$ & $\mathrm{~B}$ & A & $\mathrm{D}$ & $\mathrm{C}$ & $\mathrm{C}$ & B & B & $\mathrm{D}$ & $\mathrm{C}$ & A & $\mathrm{C}$ & B & $\mathrm{D}$ & B & 2 & INSUFICIENTE \\
\hline 4 & $\mathrm{~F}$ & $\mathrm{D}$ & $\overline{\mathrm{D}} \mathrm{I}$ & $\mathrm{B}$ & \begin{tabular}{|l|}
$\mathrm{C}$ \\
\end{tabular} & $\overline{\mathrm{A}}$ & B & $\mathrm{D}$ & $\overline{\mathrm{A}}$ & $\mathrm{D}$ & $\bar{C}$ & $\bar{C}$ & $\bar{C}$ & A & $\bar{C}$ & $\bar{C}$ & $\mathrm{D}$ & 9 & $\overline{\mathrm{BOM}}$ \\
\hline 13 & $\mathrm{~F}$ & D & $\mathrm{B}$ & $\mathrm{A}$ & C & 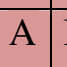 & $\mathrm{B}$ & C & B & $\mathrm{A}$ & $\mathrm{D}$ & C & $\mathrm{C}$ & B & $\mathrm{A}$ & $\mathrm{C}$ & $\mathrm{D}$ & 7 & $\mathrm{BOM}$ \\
\hline 16 & $\mathrm{M}$ & \begin{tabular}{|l|l|}
$\mathrm{A}$ \\
\end{tabular} & $\mathrm{D}$ & B & C & $\mathrm{D}$ & $\mathrm{D}$ & B & $\mathrm{D}$ & $\mathrm{D}$ & $\mathrm{C}$ & A & B & $\mathrm{D}$ & $\mathrm{C}$ & A & A & 7 & $\mathrm{BOM}$ \\
\hline 2 & $\mathrm{~F}$ & B & $\overline{\mathrm{C}}$ & $\mathrm{A}$ & \begin{tabular}{|l|l|}
$A$ \\
\end{tabular} & $\begin{array}{ll}\mathrm{A} & 1 \\
\end{array}$ & $\mathrm{~B}$ & $\mathrm{D}$ & $\bar{D}$ & $\mathrm{D}$ & $\bar{C}$ & $\bar{C}$ & $\bar{A}$ & $\bar{D}$ & $\mathrm{C}$ & $\bar{B}$ & $\bar{A}$ & 10 & MUITO BOM \\
\hline 3 & $\mathrm{~F}$ & B & $\mathrm{B}$ & $\mathrm{A}$ & $\mathrm{D}$ & $\mathrm{A}$ & B & B & A & $\mathrm{D}$ & $\mathrm{D}$ & $\mathrm{C}$ & C & A & $\mathrm{C}$ & B & B & 12 & MUITO BOM \\
\hline 9 & $\mathrm{~F}$ & B & $\mathrm{D}$ & B & $\mathrm{C}$ & A & B & B & A & $\mathrm{D}$ & $\mathrm{D}$ & $\mathrm{C}$ & $\mathrm{C}$ & A & $\mathrm{C}$ & $\mathrm{C}$ & $\mathrm{D}$ & 12 & MUITO BOM \\
\hline 11 & M & A & $\mathrm{D}$ & $\mathrm{A}$ & C & $\mathrm{D}$ & $\mathrm{A}$ & B & A & $\mathrm{D}$ & $\mathrm{D}$ & $\mathrm{C}$ & $\mathrm{C}$ & $\mathrm{D}$ & $\mathrm{C}$ & $\mathrm{C}$ & A & 12 & MUITO BOM \\
\hline 12 & $\mathrm{~F}$ & B & $\mathrm{C}$ & $\mathrm{A}$ & $\mathrm{D}$ & $\mathrm{A}$ & B & $\mathrm{D}$ & A & $\mathrm{D}$ & $\mathrm{C}$ & $\mathrm{C}$ & $\mathrm{C}$ & D & B & B & $\mathrm{D}$ & 10 & MUITO BOM \\
\hline
\end{tabular}

\begin{tabular}{|c|c|c|c|c|c|}
\hline Afirmativas & $\begin{array}{l}\text { Com muita } \\
\text { dificuldade }\end{array}$ & $\begin{array}{l}\text { Com pouca } \\
\text { dificuldade }\end{array}$ & $\begin{array}{l}\text { Nem } \\
\text { facilidade, } \\
\text { nem } \\
\text { dificuldade }\end{array}$ & $\begin{array}{l}\text { Com } \\
\text { pouca } \\
\text { facilidade }\end{array}$ & $\begin{array}{l}\text { Com } \\
\text { muita } \\
\text { facilidade }\end{array}$ \\
\hline Eu entendo gráficos e tabelas & & & $\begin{array}{l}5,14 \\
13,2,11\end{array}$ & 16,9 & $4,3,12$ \\
\hline $\begin{array}{l}\text { Consigo escolher palavras } \\
\text { chave de textos/conteúdos }\end{array}$ & & & $4,9,11$ & $\begin{array}{l}5, \\
14,13,16,2, \\
3,12,\end{array}$ & \\
\hline $\begin{array}{l}\text { Consigo perceber semelhanças } \\
\text { e diferenças entre textos ou } \\
\text { gráficos }\end{array}$ & & & 12 & & $\begin{array}{l}5,14,4 \\
13,16 \\
2,3,9,11\end{array}$ \\
\hline $\begin{array}{l}\text { Sei distinguir entre } \\
\text { informações importantes ou } \\
\text { não para resolver problemas }\end{array}$ & & $14,4,2$, & 13, & 5,16 & $3,9,11,12$ \\
\hline Consigo interpretar textos & & $4,13,9$ & 11, & 2 , & $\begin{array}{l}5,14,16,3, \\
12\end{array}$ \\
\hline Consigo justificar respostas & 13 , & & & $5,2,9$ & $\begin{array}{l}14,4 \\
6,3,11,12\end{array}$ \\
\hline
\end{tabular}

Tabela - Como estudantes se auto-avaliaram em relação a habilidades requeridas na resolução de problemas. Os números são os códigos dos alunos.

\section{SOBRE AS AUTORAS}

RAQUEL RUPPENTHAL. Licenciada em Ciências Biológicas pela Universidade Federal de Santa Maria (UFSM). Especialista em Mídias na Educação e Tecnologias da Informação e Comunicação aplicadas a educação. Mestre em Educação em Ciências (UFSM). Doutoranda em Educação em Ciências (UFSM). Professora na rede estadual do Rio Grande do Sul. 
MARIA ROSA CHITOLINA SCHETINGER. Licenciada em Biologia pela Universidade Federal do Rio Grande do Sul (UFRGS). Mestre e doutora em Bioquímica (UFRGS). Professora da Universidade Federal de Santa Maria.

Recebido: 15 de junho de 2016.

Revisado: 27 de novembro de 2016.

Aceito: 24 de janeiro de 2017. 